\title{
A Combinational Approach Using Matrix Based Pairwise Key Establishment and Post Deployment Approach in Wireless Sensor Networks
}

\author{
Dinesh Dhakar $^{1}$, Ravi Khatri ${ }^{2}$ \\ ${ }^{1}$ PG Student, Department of CSE, VITM, Indore (M.P), India \\ ${ }^{2}$ Associate Professor, Department of CSE, VITM, Indore (M.P), India
}

\begin{abstract}
Key distribution schemes ahs always played a pivotal role in the security of wireless sensor networks. In this research work we focus mainly on the security aspect of WSN. We have developed a modified key distribution scheme which uses the concepts of post as well as pre distribution scheme and thus hs proved to be a better alternative then the rest of two schemes. Simulation study has been carried out using matlab. The effort turned out to be fruitful s our modified scheme showed less dead nodes per round of data transfer as compared to post deployment scheme.
\end{abstract}

Keywords: Security, Key establishment, Mobile sensor networks, Key prioritization, Post-deployment knowledge.

\section{Introduction}

Distributed sensor networks have received a lot of attention recently due to its wide applications in military as well as civilian operations. Example applications include target tracking, scientific exploration, and data acquisition in hazardous environments. The sensor nodes are typically small, low-cost, battery powered, and highly resource constrained. They usually communicate with each other through wireless links. Security services such as authentication and key management are critical to secure the communication between sensor nodes in hostile environments. As one of the most fundamental security services, pairwise key establishment enables the sensor nodes to communicate securely with each other using cryptographic techniques. However, due to the resource constraints on sensor nodes, it is not feasible for them to use traditional pairwise key establishment techniques such as public key cryptography and key distribution center (KDC).

Instead of the above two techniques, sensor nodes may establish keys between each other through key pre distribution, where keying materials are pre distributed to sensor nodes before deployment. As two extreme cases, one may setup a global key among the network so that two sensor nodes can establish a key based on this global key, or assign each sensor node a unique random key with each of the other nodes. However, the former is vulnerable to the compromise of a single node, and the latter introduces huge storage overhead on sensor nodes.

Eschenauer and Gligor proposed a probabilistic key predistribution scheme recently for pairwise key establishment [Eschenauer and Gligor 2002]. The main idea is to let each sensor node randomly pick a set of keys from a key pool before the deployment so that anytwo sensor nodes have a certain probability to share at least one common key. Chan et al. further extended this idea and developed two key predistribution techniques: a q-composite key pre distribution scheme and a random pairwise keys scheme
[Chan et al. 2003]. The q -composite key predistribution also uses a key pool but requires two nodes compute a pairwise key from at least q predistributed keys that they share. The random pairwise keys scheme randomly picks pairs of sensor nodes and assigns each pair a unique random key. Both schemes improve the security over the basic probabilistic key predistribution scheme. However, the pairwise key establishment problem is still not fully solved. For the basic probabilistic and the $\mathrm{q}$-composite key predistribution schemes, as the number of compromised nodes increases, the fraction of affected pairwise keys increases quickly. As a result,a small number of compromised nodes may affect a large fraction of pairwise keys. Though the random pairwise keys scheme does not suffer from the above security problem, given a memory constraint, the network size is strictly limited by the desired probability that two sensor nodes share a pairwise key, the memory available for keys on sensor nodes, and the number of neighbor nodes that a sensor node can communicate with. In this paper, we develop a number of key predistribution techniques to deal with theabove problems. We first develop a general framework for pairwise key establishmentbased on the polynomial-based key predistribution protocol in [Blundo et al. 1993] and the probabilistic key distribution in [Eschenauer and Gligor 2002; Chan et al. 2003]. This framework is called polynomial pool-based key predistribution, which uses a polynomialpool instead of a key pool in [Eschenauer and Gligor 2002; Chan et al. 2003]. The secrets on each sensor node are generated from a subset of polynomials in the pool. If two sensor nodes have the secrets generated from the same polynomial, they can establish a pairwise key based on the polynomial-based key predistribution scheme. All the previous schemesin [Blundo et al. 1993; Eschenauer and Gligor 2002; Chan et al. 2003] can be considered as special instances in this framework.

By instantiating the components in this framework, we further develop two novel pair-wise key pre distribution schemes: a random subset assignment scheme and a hypercube-based scheme. The random subset assignment scheme assigns each sensor node the secrets generated from

\section{Volume 4 Issue 11, November 2015}




\section{International Journal of Science and Research (IJSR) \\ ISSN (Online): 2319-7064}

Index Copernicus Value (2013): 6.14 | Impact Factor (2014): 5.611

a random subset of polynomials in the polynomial pool. The hypercube-based scheme arranges polynomials in a hypercube space, assigns each sensor node to a unique coordinate in the space, and gives the node the secrets generated from the polynomials related to the corresponding coordinate. Based on this hypercube, each sensor node can then identify whether it can directly establish a pairwise key with another node, and if not, what intermediate nodes it can contact to indirectly establish the pairwise key. Our analysis indicates that our new schemes have some nice features compared with the previous methods. In particular, when the fraction of compromised secure links is less than $60 \%$, given the same storage constraint, the random subset assignment scheme provides a significantly higher probability of establishing secure communication between noncompromised nodes than the previous methods. Moreover, unless the number of com-promised nodes sharing a common polynomial exceeds a threshold, compromise of sensor nodes does not lead to the disclosure of keys established between non-compromised nodes using this polynomial.

Similarly, the hypercube-based scheme also has a number of attractive properties. First, it guarantees that any two nodes can establish a pairwise key when there are no compromised nodes, provided that the sensor nodes can communicate with each other. Second, it is resilient to node compromise. Even if some sensor nodes are compromised, there is still a high probability to re-establish a pairwise key between noncompromised nodes. Third, a sensor node can directly determine whether it can establish a pairwise key with another node and how to compute the pairwise key if it can. As a result, there is no communication overhead during the discovery of directly shared keys. Evaluation of polynomials is essential to the proposed schemes, since it affects the performance of computing a pairwise key. To reduce the computation at sensor nodes, we provide an optimization technique for polynomial evaluation. The basic idea is to compute multiple pieces of key fragments over some special finite fields such as F28 +1 and F216 +1 and concatenate these fragments into a regular key. A nice property provided by such finite fields is that no division is necessary for modular multiplication. As a result, evaluation of polynomials can be performed efficiently on low cost processors on sensor nodes that do not have division instructions. Our analysis indicates that such a method only slightly decreases the uncertainty of the keys.

\section{Implementation of Key Predistribution Scheme}

The basic algorithms for key predistribution scheme for matrix based system can be written as below:-

1) Choose ' $N$ ' independent key seeds designated as $s_{1}, s_{2}$, $\mathrm{S}_{3}, \ldots . . \mathrm{s}_{\mathrm{N}}$.

2) Let their id's be $\mathrm{id}_{1}, \mathrm{id}_{2}, \mathrm{id}_{3}, \mathrm{id}_{4}, \mathrm{id}_{5}, \mathrm{id}_{\mathrm{N}}$

3) Consider a matrix $h$ as per [1].

4) Create a lambda $x$ lambda matrix as per [1].

5) Calculate matrix $A$ as per [1].

6) Generate keys and broadcast keys to each node.

7) Each node will then transmit packets to $\mathrm{BS}$ via other nodes according to matrix $\mathrm{A}$ and with the help of keys stored.

8) Because keys are distributed and broadcasted to each nodes in advance this scheme is called as key pre distribution.

9) This scheme has been implemented for 100 nodes and for 100 rounds of data packets and the results are as below :-

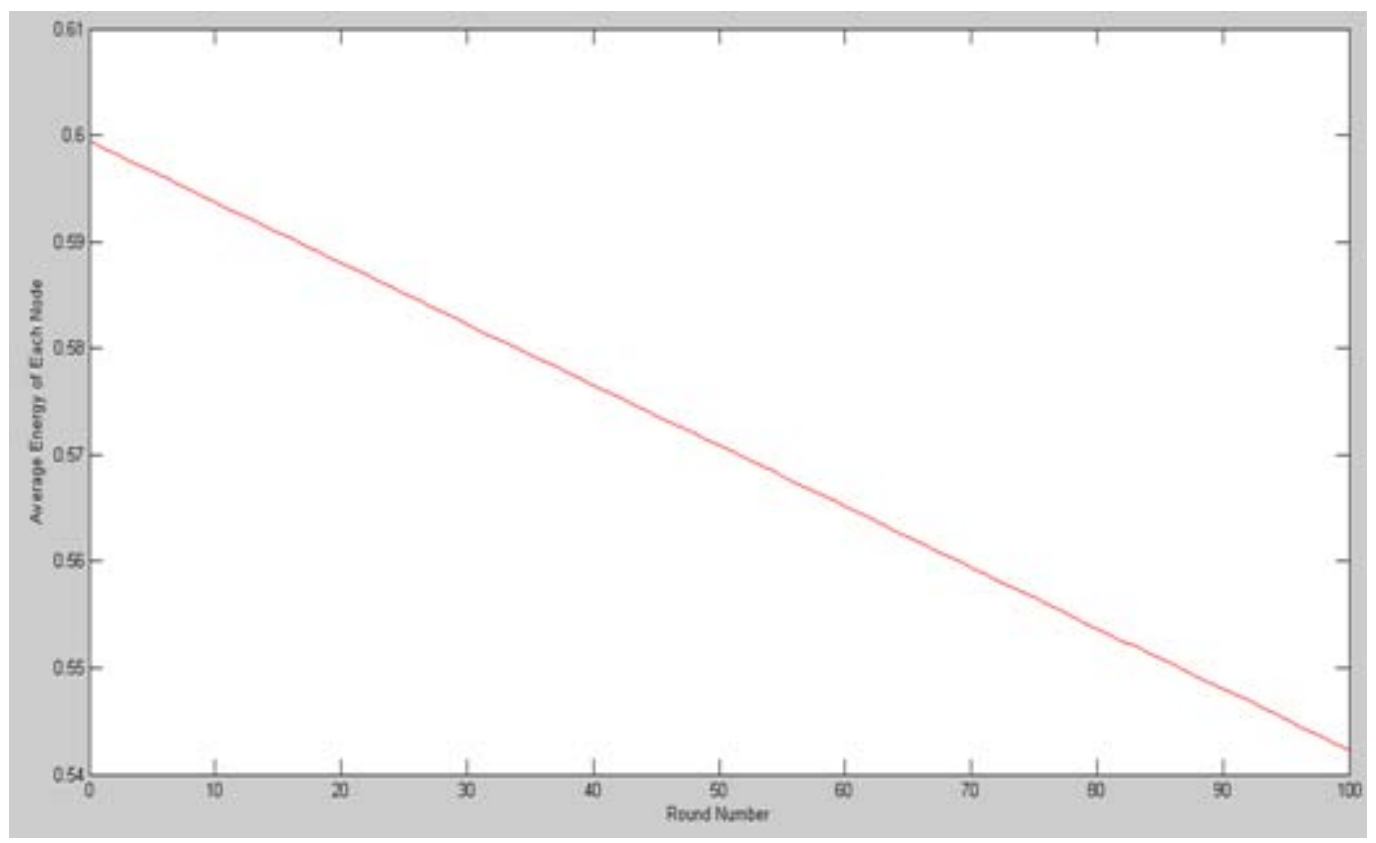

Figure 5.1: Average energy spent per round for key predistribution scheme 


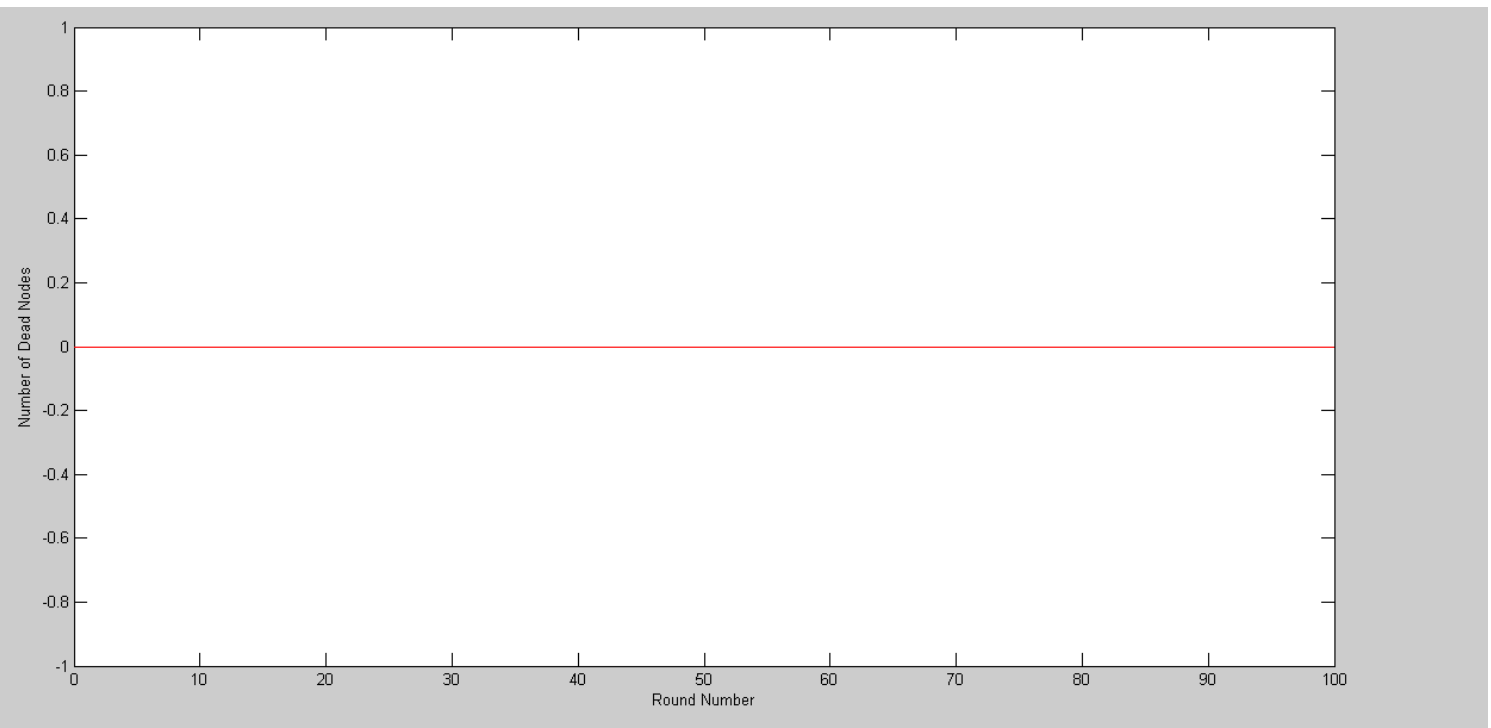

Figure 5.2: No. of dead nodes per round for key predistribution scheme

\section{Implementation of Post Deployment Analysis}

In this method as per given in [2] basic steps are as below:

1) ' $m$ ' key units are generated by the system in a set of ' $M$ ' , such that each node can store a maximum of ' $\mathrm{m}$ ' key units.

2) A unique id is assigned to each node.

3) Each ' $m$ ' key units are randomly distributed to each node.

4) Then nodes are deployed physically and their locations are determined using gps and this information is called as their unique location.

5) Prior to distributing keys the locations are also determined randomly and associated with each node.

6) Then each node will determine the distance between other nodes and then the key will be shared as per [2].

7) Because the keys are not broadcasted as shown in [1] this scheme is referred to a post deployment analysis.

The results for this algorithm in terms of energy and dead nodes per round are given as below:-

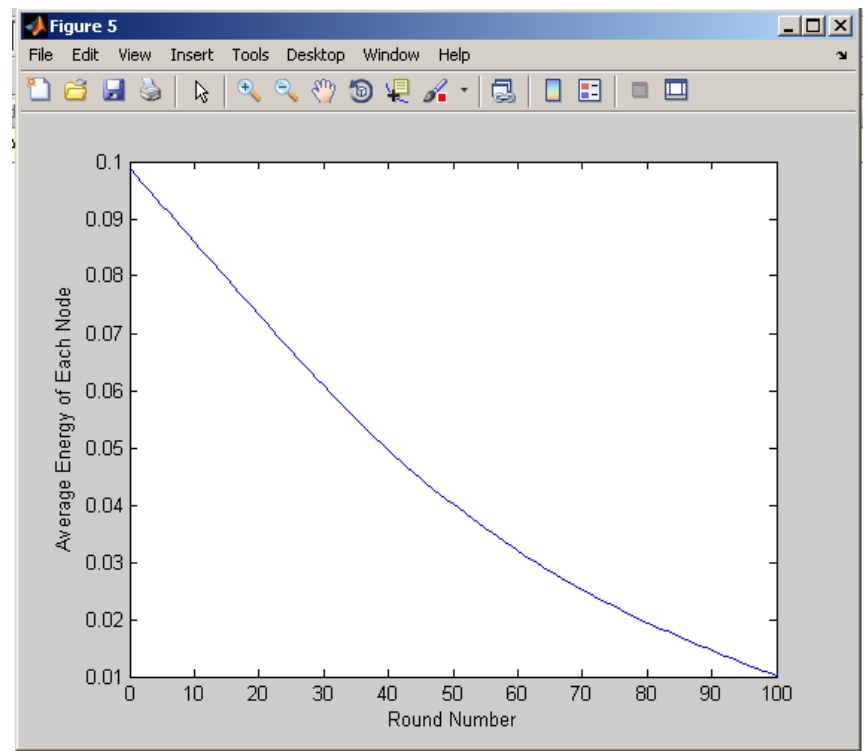

Figure 5.3: Average energy spent per round for post deployment scheme

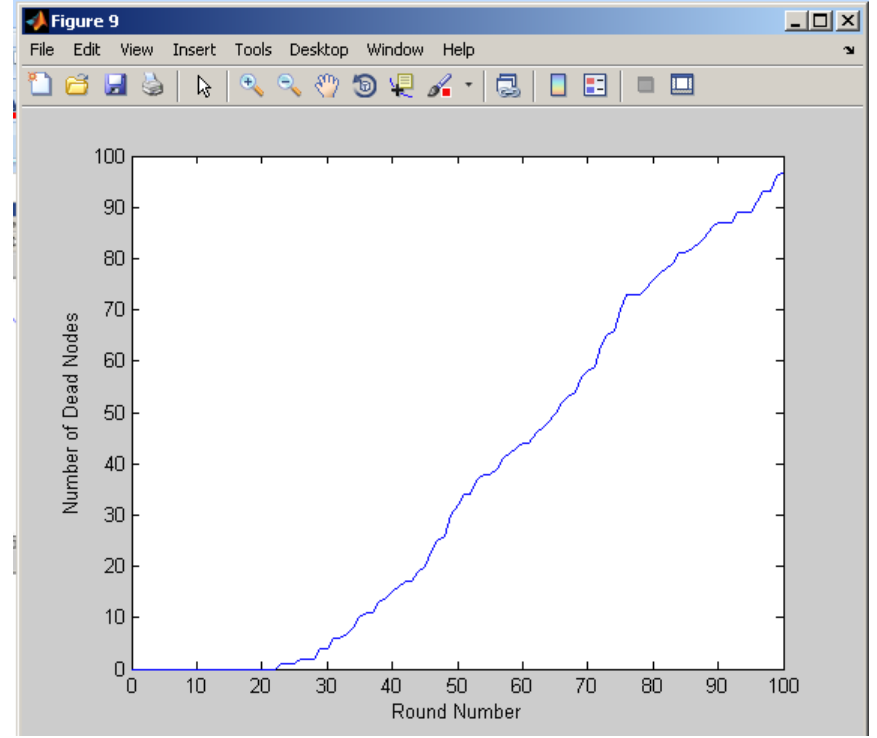

Figure 5.4: No. of dead nodes per round for post deployment scheme

\section{A Combinational Approach}

In our work we have combined the benefits of the above two schemes and then simulated the entire setup for 100-500 rounds of data transfer. The basic steps involved in our approach are as follows:-

1) We have assumed that the position of nodes are not determined in advance contrary to pre distribution scheme and thus the locations of the nodes are determined via gps but this time the information is relayed to base station.

2) The base station then depending on the location of each node will broadcast the key matrix to each node as per in [1].

3) This distributed common matrix will be used by all the nodes to generate further key for communication.

4) Each node then will determine the distance between other nodes as per [2].

5) This information is then used for effective communication i.e the node will not broadcast the information, rather the information will be relayed form 


\section{International Journal of Science and Research (IJSR) \\ ISSN (Online): 2319-7064}

Index Copernicus Value (2013): 6.14 | Impact Factor (2014): 5.611

one node to another as in hierarchical wireless sensor network.

6) The LEACH clustering algorithm has been deployed for further data transfer.

7) Because the information is relayed form one node to another the overall dead node occurrence is significantly reduced and hence the data transfer gets complete without overloading the nodes.

Various simulation results for the said scheme are as given below:-

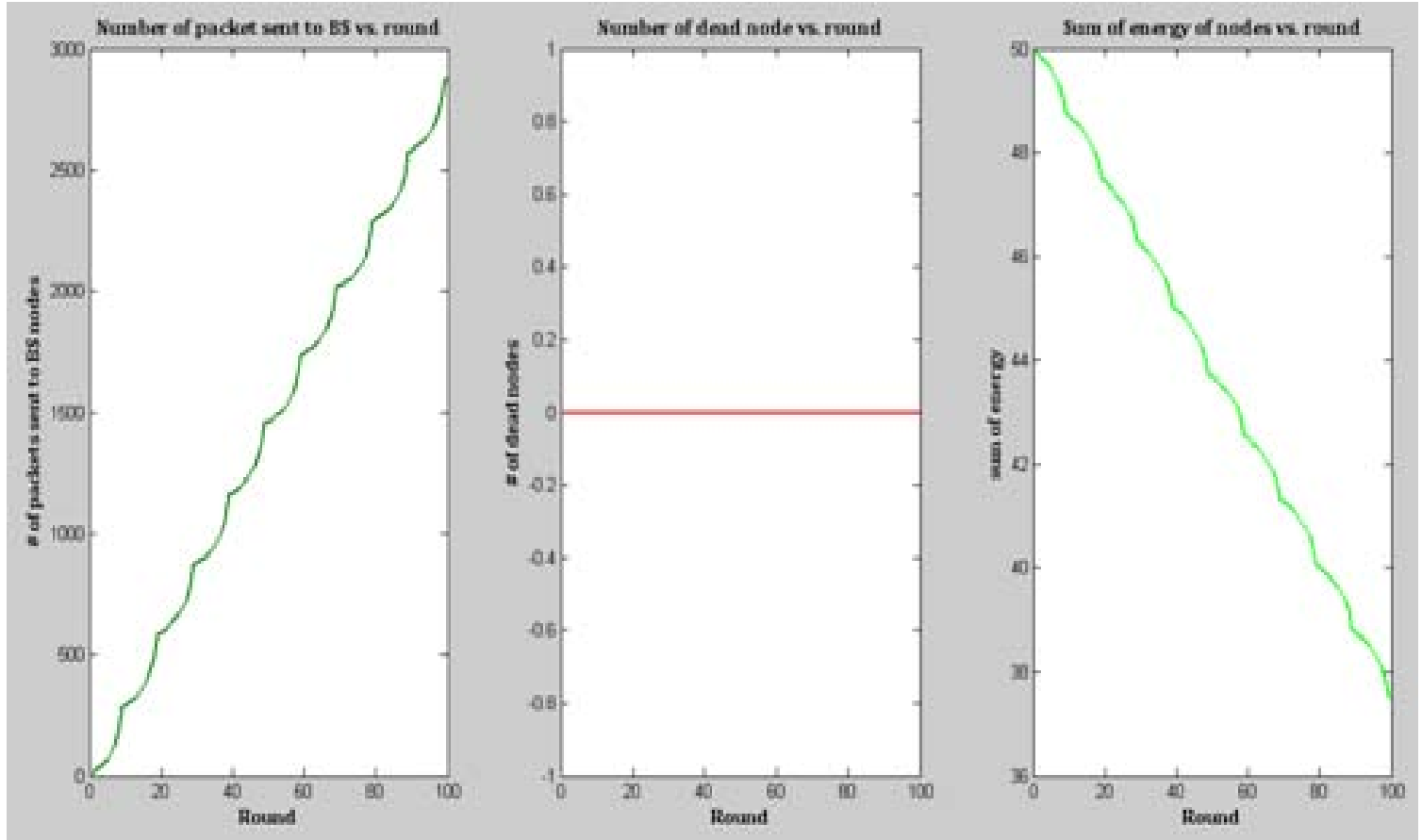

Figure 5.5: Simulations results for modified algorithms for 100 rounds
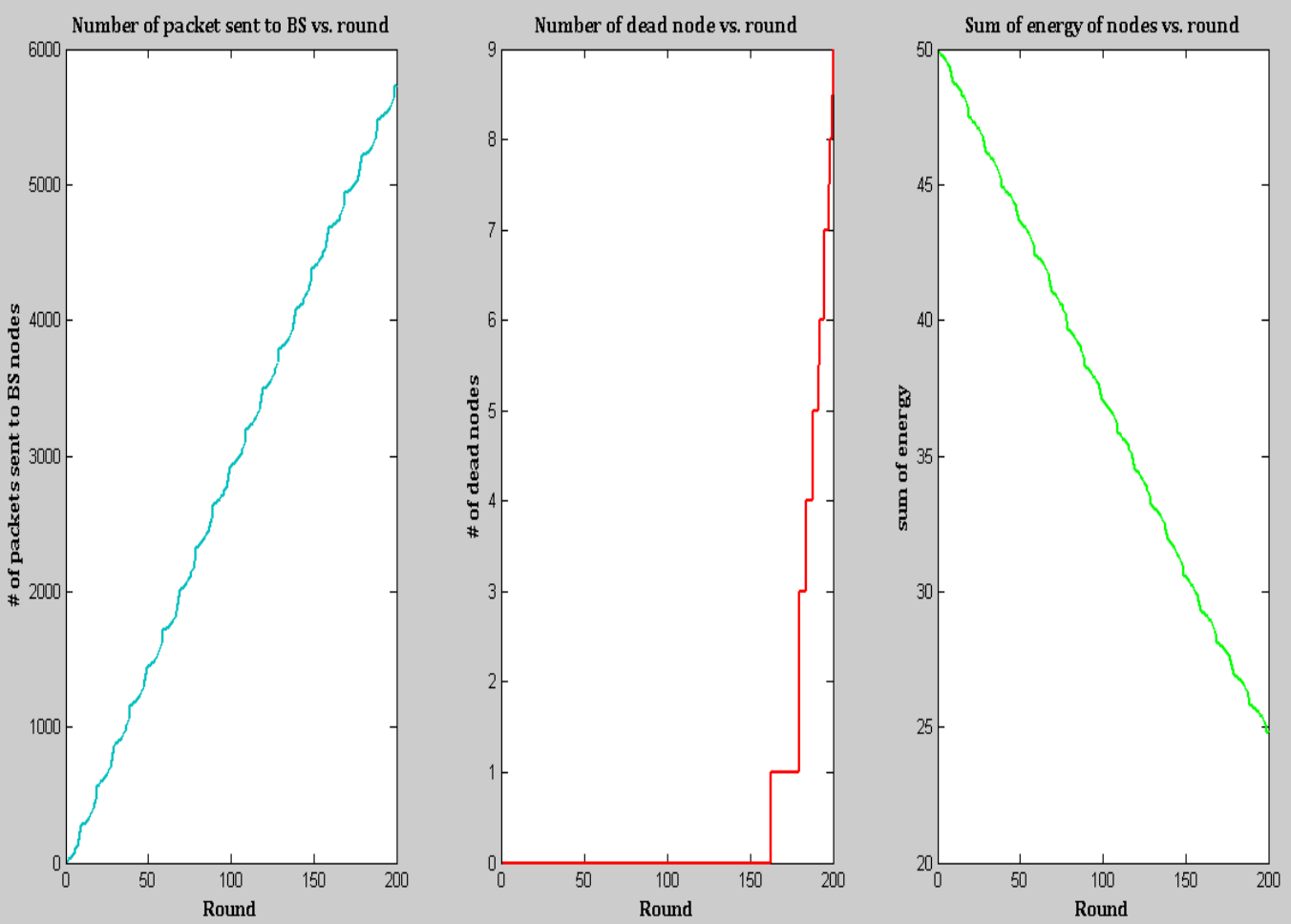

Figure 5.6: Simulations results for modified algorithms for 200 rounds 
International Journal of Science and Research (IJSR)

ISSN (Online): 2319-7064

Index Copernicus Value (2013): 6.14 | Impact Factor (2014): 5.611
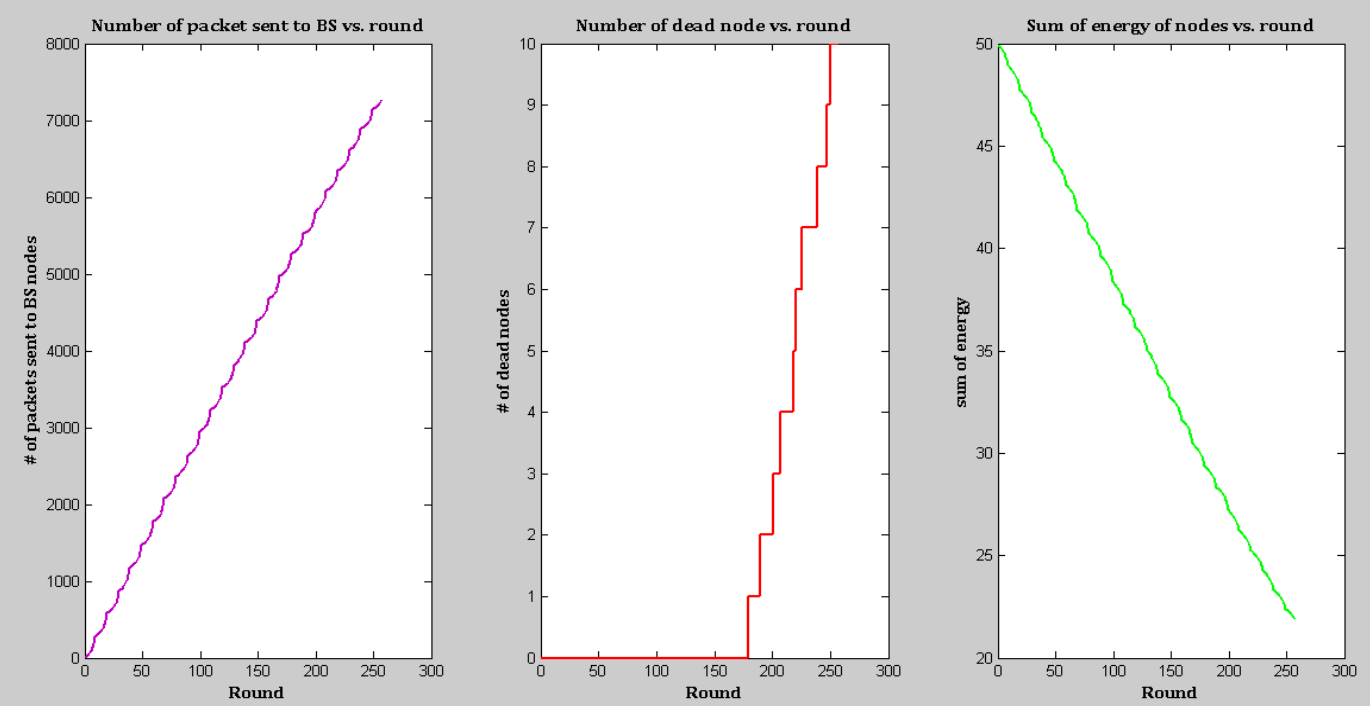

Figure 5.6: Simulations results for modified algorithms for 300 rounds
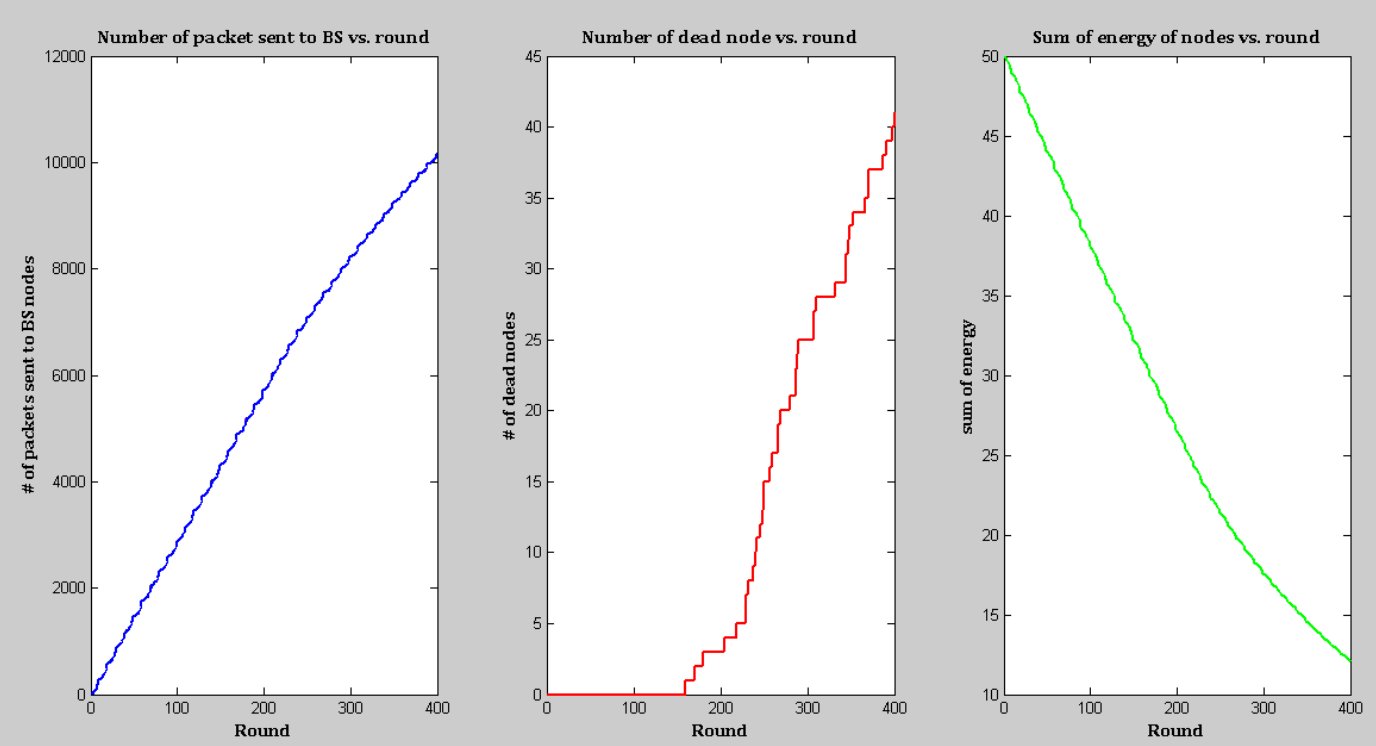

Figure 5.7: Simulations results for modified algorithms for 400 rounds
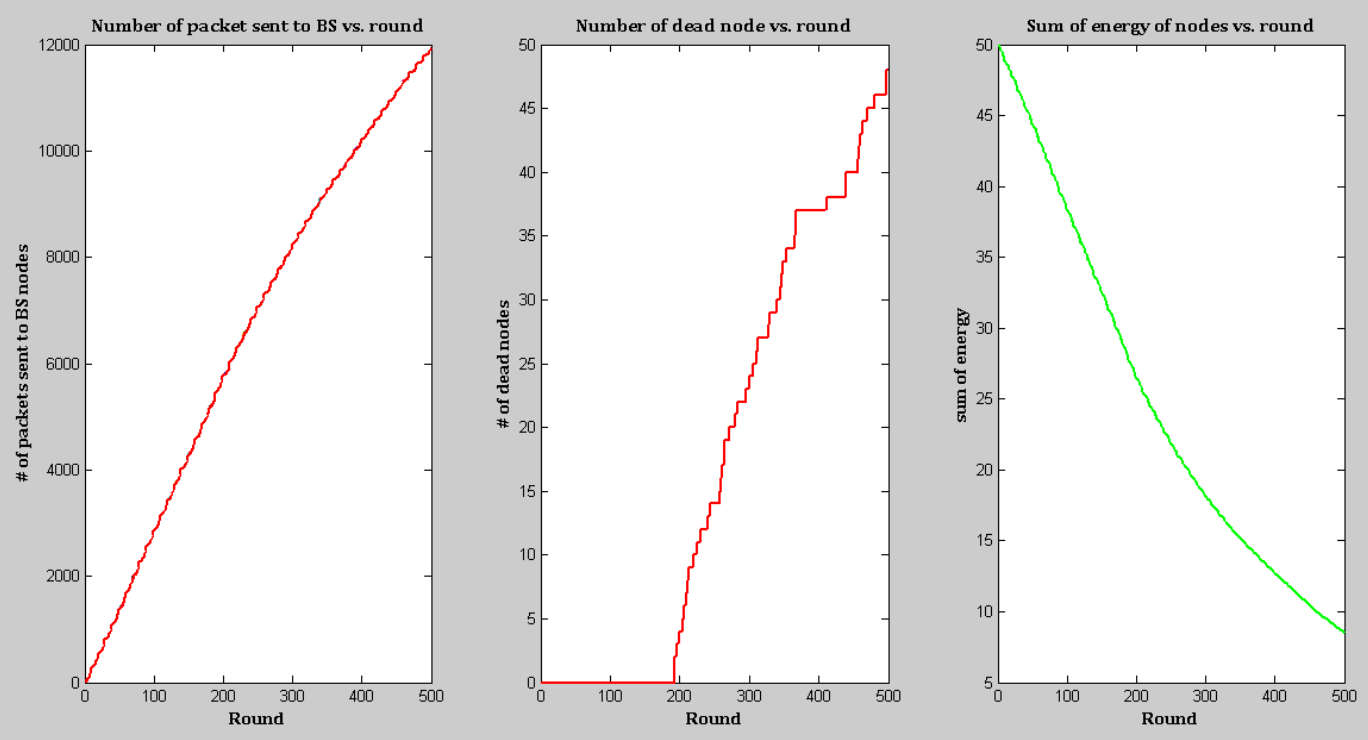

Figure 5.8: Simulations results for modified algorithms for 500 rounds

Volume 4 Issue 11, November 2015

www.ijsr.net 


\section{International Journal of Science and Research (IJSR) \\ ISSN (Online): 2319-7064}

Index Copernicus Value (2013): 6.14 | Impact Factor (2014): 5.611

\section{Conclusion}

In this research work, we developed a general framework for polynomial pool-based pairwise key predistribution in sensor networks based on the basic polynomial-based key predistribution in [1]. This framework allows study of multiple instantiations of possible pairwise key establishment schemes. Based on this framework, we developed two specific key predistribution schemes: the random subset assignment scheme and the hypercube-based key predistribution scheme. Our analysis of these schemes indicate that both schemes have significant advantages over the existing approaches. The implementation and experimental results also demonstrate the practicality and efficiency in the current generation of sensor networks. Several research directions are worth investigating. First, we observe sensor node have low mobility in many applications. Thus, it may be desirable to develop locationsensitive key predistribution techniques to improve the probability for neighbor nodes to share common keys and at the same reduce the threat of compromised nodes. Second, it is critical to detect and/or revoke compromised nodes from an operational sensor network.

It has been shown in the result analysis that the post deployment analysis scheme given in [2] has major drawbacks in terms of no of dead nodes per round of data transfer, our results are better then both the approaches but several mathematical models are still need to be made, thus this work has to completed in the future to avoid any disambiguousness in the research literature.

\section{References}

[1] Wireless Integrated Network Sensors, University ofCalifornia, http://www.janet.ucla.edu/WINS.

[2] I. F. Akyildiz, W. Su, Y. Sankarasubramaniam, andE. Cayirci. A survey on sensor networks. IEEE Communications Magazine, 40(8):102-114, August 2002[3] R. Anderson and M. Kuhn. Tamper resistance a cautionarynote. In Proceedings of the Second Usenix Workshop onElectronic Commerce, pages 1-11, November 1996.

[3] R. Blom. An optimal class of symmetric key generationsystems. Advances in Cryptology: Proceedings ofEUROCRYPT 84 (Thomas Beth, Norbert Cot, and IngemarIngemarsson, eds.), Lecture Notes in Computer Science,Springer-Verlag, 209:335338, 1985.

[4] C. Blundo, A. D. Santis, A. Herzberg, S. Kutten, U. Vaccaro,and M. Yung. Perfectly-secure key distribution for dynamicconferences. Lecture Notes in Computer Science, 740:471-486, 1993.

[5] D. W. Carman, P. S. Kruus, and B. J. Matt. Constraints andapproaches for distributed sensor network security. NAI LabsTechnical Report \#00-010, available athttp://download.nai.com/products/media/nai/zip/naila bs-report-00-010-final.zip,2000.

[6] H. Chan, A. Perrig, and D. Song.Random keypredistribution schemes for sensor networks. In IEEESymposium on Security and Privacy, pages 197213,Berkeley, California, May 11-14 2003.
[7] W. Diffie and M. E. Hellman.New directions incryptography. IEEE Transactions on Information Theory,22:644-654, November 1976.

[8] W. Du, J. Deng, Y. S. Han, S. Chen, and P. K. Varshney. Akey management scheme for wireless sensor networks usingdeployment knowledge. Technical Report, SyracuseUniversity, July 2003. Available

fromhttp://www.cis.syr.edu/ wedu/Research/paper/ddh cv03.pdf.

[9] Erd"os and R'enyi. On random graphs I. Publ. Math.Debrecen, 6:290-297, 1959.

[10]L. Eschenauer and V. D. Gligor.A key-management scheme for distributed sensor networks. In Proceedings of the $9^{\text {th }} \mathrm{ACM}$ conference on Computer and communications security, November 2002.

[11] J. M. Kahn, R. H. Katz, and K. S. J. Pister. Next centurychallenges: Mobile networking for smart dust. InProceedings of the 5th Annual ACM/IEEE InternationConference on Mobile Computing and Networking(MobiCom), pages 483-492, 1999.

[12]F. J. MacWilliams and N. J. A. Sloane.The Theory ofError-Correcting Codes. New York, NY: Elsevier Science Publishing Company, Inc., 1977.

[13]D. Malkhi, M. Reiter, A. Wool, and R. N. Wright. Probabilistic quorum systems. Information and Computation, (2):184-206, November 2001.

[14]B. C. Neuman and T. Tso. Kerberos: An authentication service for computer networks. IEEE Communications,32(9):33-38, September 1994.

[15] A. Perrig, R. Szewczyk, V. Wen, D. Cullar, and J. D. Tygar.SPINS: Security protocols for sensor networks. In Proceedings of the 7th Annual ACM/IEEE Internation Conference on Mobile Computing and Networking (MobiCom) , pages 189-199, Rome, Italy, July 2001. 